\title{
Evaluation of the Lobelia inflata Extract in the Histopathological Profile of Melanoma in Experimental Model
}

\author{
Layene C Ireno $^{1}$, Nathalia P Garcia ${ }^{1}$, Mirella RA Moreira ${ }^{2}$, Marcello P Castro ${ }^{1}$, Karina F Zoccal ${ }^{1}$ \\ and Cristiane Tefé-Silval*1
}

${ }^{1}$ Barão de Mauá University Center, Brazil

${ }^{2}$ Department of Clinical Analyzes, Toxicology and Bromatology, School of Pharmaceutical Sciences of Ribeirão Preto, University of São Paulo, Brazil

*Corresponding author: Cristiane Tefé-Silva, University Center Barão de Mauá, Brazil

\section{ARTICLE INFO}

Received: 唪 February 25, 2020

Published: 豐 March 06, 2020

Citation: Layene C I, Nathalia P G, Mirella RA M, Marcello P C, Cristiane Tefé-Silva, et al., Evaluation of the Lobelia inflata Extract in the Histopathological Profile of Melanoma in Experimental Model. Biomed J Sci \& Tech Res 26(3)-2020. BJSTR. MS.ID.004347.

\begin{abstract}
Melanoma is a type of skin cancer extremely aggressive affecting more than one thousand people in the world. Late stages and widely spread malignant melanoma do not respond to standard therapeutic approaches. Therefore, in this study we induced the injecting primary tumor with melanoma B16F10 cells in mice and treated the animals with Lobelia inflata for 22 days to evaluate the antitumor potential of the plant extract. The animals were monitored, the skin and cutaneous tumor were excised, and the tissues were prepared for histopathological and morphometric analyses. Regarding to microscopic aspects, there was a reduction in the angiogenesis process ratified by the reduction of the number of blood vessels after interaction with the extract and, in addition, part of the chronic inflammation, reflected by the number of macrophages decreased after treatment of the mice that received the extract.
\end{abstract}

Keywords: Melanoma; Lobelia inflata; Antitumoral Treatment; Skin Cancer; Alternative Plants

\section{Introduction}

Cutaneous melanoma is a malignant neoplasm with a multifactorial etiology, characterized by the abnormality of melanocytes that invade the basement membrane. Neoplastic cells use proteins produced by fibroblasts that also release growth factors that melanoma is not able to synthesize. These growth factors can increase the growth capacity and invasion [1]. This is one of the most aggressive neoplasms, predominantly among the white population due to its greater sensitivity to sun exposure [2]. It is a tumor recognized by elevated mortality due to its high metastatic, high aggressiveness and low response to conventional therapies. In spite of corresponding to $4 \%$ of the neoplasias, cutaneous melanoma is responsible for $80 \%$ of deaths being thus considered a serious public health problem [3]. The earlier the lesions are, the greater the chance of cure, but the risk of death grows in proportion to the increase of the thickness of the tumor [4]. On average, patients who are at late-stage melanoma survive from 6 to 10 months [5]. Distinctly from the disease diagnosed at the initial treatment, which is effectively treated with surgical, the metastatic stage is almost universally fatal, with the response rate to chemotherapy treatment being less than $30 \%$ of cases [6].

Despite its seriousness, the available therapeutic for the treatment of this tumor often results in unsatisfactory results. Since advanced melanoma does not respond rapidly towards standard therapies such as chemotherapy, radiotherapy and surgical excision, the search for new anti-melanoma agents is of an urgent need. In this regard, studies have been developed seeking for alternative therapies through the use of medicinal plant-based products for the treatment of melanoma. It is known that Lobelia inflata is a plant belonging to the family Campanulaceae, known as Indian tobacco, popularly widely used because of its emetic, hypnotic, anti-asthmatic, astringent and tobacco substitute properties in alternative therapies. This species is a source of alkaloid 
substances that motivated studies since its isolation in 1885, when its therapeutic properties was thought to be understood [7]. There are a lot of other substances isolated in the genus Lobelia such as piperidine alkaloids including lobeline, lobelane, lobelanidine, norlobelanine, and lobelanine. Besides, flavonoids, terpenes and triterpenes, saponins, and coumarins have also been identified and probably have pharmacological effects.

Among the cited species of genus, Lobelia inflata is better known and contains more than 20 piperidine alkaloids, being the subject of much research regarding the properties of its extract. On the other hand, another species, Lobelia chinensis, has also become the focus of several studies and explained literature that has known antitumor activities in the treatment of breast and stomach tumors, as well as hemostatic, diuretic and anti- inflammatory characteristics. Some flavonoids are specially found in Lobelia chinensis and are responsible to modulate key cellular enzyme function, acting as anti-cancer, anti-mutagenic, antioxidant and anti-inflammatory substance [8]. However, little is known about the antitumor effect of Lobelia inflata on growth and development of melanomas. It is necessary to discover and understand the probable mechanism of action involved in the interaction between Lobelia inflata and cutaneous melanoma. Thus, upon these considerations, in the present work, we took advantage of B16F10 melanoma cells, which is a well validated model for both primary tumor and we investigated the antitumoral activity of Lobelia inflata during melanoma progression.

\section{Materials and methods}

\section{Animal Care and Ethics Statement}

Balb/c mice (6-8 weeks old), weighing approximately 20g, were bred and maintained at $25^{\circ} \mathrm{C}$, with $12 \mathrm{~h} / 12 \mathrm{~h}$ dark/light cycle in the animal facilities of the University Center Barão de Mauá, fed with laboratory chow, and given tap water to drink ad libitum. All procedures were approved by the Ethics Committee on Research and Animal Experimentation of University Center Barão de Mauá, under protocol n.253/16.

\section{Experimental Design}

Balb/c mice ( $n=7 /$ experimental group) were divided into four groups:

Group 01- PBS (phosphate buffered saline): control

Group 02- Lobelia: Water + treatment with Lobelia inflata extract.

Group 03- Melanoma: received inoculation of B16F10 and PBS cells.

Group 04- Melanoma + Lobelia: received inoculation of B16F10 cells and Lobelia inflata extract treatment.
One day before the implant, the mice were trichotomized in the anterior dorsal region. On the day of the experiment, a suspension containing $10^{6}$ cells /mL of B16F10 murine melanoma cells was implanted subcutaneously into the back of each mouse in groups 03 (Melanoma) and 04 (Melanoma + Lobelia). Treatment was performed orally every five days over a total of 20 days (thus applied on days $0,5,10,15,20)$. The animals received Lobelia inflate or water. Experimental groups were killed 22 days after inoculation of B16F10 cells in a $\mathrm{CO}_{2}$ chamber.

\section{Cell Culture}

B16F10 melanoma cells were cultured in culture medium and, once confluent, in the exponential growth phase, were counted and adjusted to the concentration of $10^{6}$ cells / $0.1 \mathrm{~mL}$ of $0.9 \%$ saline. This model is widely used in research on mice to induce melanoma by the action of a B16 derived retrovirus capable of transforming normal melanocytes into cancer cells [9].

\section{Lobelia inflata Extract}

The extract of the Lobelia inflata plant was performed as previously described [10].

\section{Animals Survival and Tumor Growth}

Tumor weight and size were monitored and measured every other day for 22 days. Tumors were measured with a Vernier caliper, and their size (in $\mathrm{mm}^{3}$ ) was calculated, as previously described [11]. Briefly, tumor volume $=$ (length $\times$ height $\times$ width $) / 2$. For this experiment, mice were monitored for 30 days after tumor injection. The macroscopic evaluation of the animals was performed daily in relation to tumor growth, according to the ABCD system, observing the possible formation of ulcers in the skin. This rule is relevant for detecting cutaneous melanoma. The letter A characterizes the asymmetry of the lesion, while the letter B means the presence of irregular borders, the letter $\mathrm{C}$ suggests color changes and the letter $\mathrm{D}$ corresponds to a tumor diameter greater than $6 \mathrm{~mm}$, being all these factors important for determining the prognosis [12]. Mortality was monitored through daily observation over the entire experimental period. Finally, the sacrifice occurred after 22 days of tumor implantation.

\section{Histopathological Evaluation}

The tumor arise in the skin was excised and the samples were immersed in $10 \%$ formaldehyde solution for 48 hours. After the fixation period, the samples were dehydrated in $80 \%, 90 \%, 95 \%$ and $100 \%$ alcohol swelling solutions, diaphanized in xylene, and thereafter waxed into paraffin. From the blocks, histological sections of $4 \mu \mathrm{m}$ thickness were made in an automatic microtome (Microtome 820 Spencer, American Optical Cooperation). The slides were kept in a greenhouse for 24 hours and stained with HE (Hematoxylin \& Eosin). 


\section{Morphometric Analysis}

Tumor morphometric analysis was performed using the ISC program, Tucsen Photonics Co., Ltd. in conjunction with a Nikon (Nikon eclipse E200) microscope, coupled to a video camera (Tucsen USB 2.0 H Series) and a computer online. The analysis was evaluated by a researcher blinded to the protocol design.

a) Number of mitoses counted from 15 randomly chosen fields in each lamina stained with HE at $200 \times$ magnification.

b) Number of vessels counted 15 fields randomly selected on each slide stained with $\mathrm{HE}$ at $40 \times$ magnification.

c) Inflammatory cells: 15 neutrophils and macrophage quantification were counted at randomly chosen fields in each lamina stained with HE at $200 \times$ magnification.

\section{Statistical Analyzes}

The Student's t-test two-tailed was applied for comparison between the groups exposed to the same stress factor (tumor). Thus, the control groups served as witnesses and were excluded from the analysis because they did not present such a factor common to the categories Melanoma and Melanoma + Lobelia. Data are expressed as mean \pm SEM (Standard Error of Mean). Differences between mean values were considered significant when $\mathrm{p}<0,05$.

\section{Results}

\section{Animal Monitoring and Tumor Growth}

In order to evaluate the contribution of Lobelia inflata extract against the growth of the melanoma, we used a model of suspension of B16F10 lineage cells in mice from one group that did not receive treatment and in another group that received the Lobelia extract. Tumor growth was monitored daily in the animals. With respect to the obtained macroscopic results, the Melanoma group was the only one whose animals developed tumor initially, with $57 \%$ of the mice developing the tumor from the beginning of the follow-up, with rapid growth especially in the last 4 days, reaching a maximum volume on day 22. In contrast, the same did not occur in the mice of the Melanoma + Lobelia group (Figure 1). Regarding ulcerations, only the melanoma group presented such alteration, considering the discontinuity of the epidermis in the tumor area, whereas in the treated group, there were no ulcerations, which suggests a better prognosis (Figure 1).

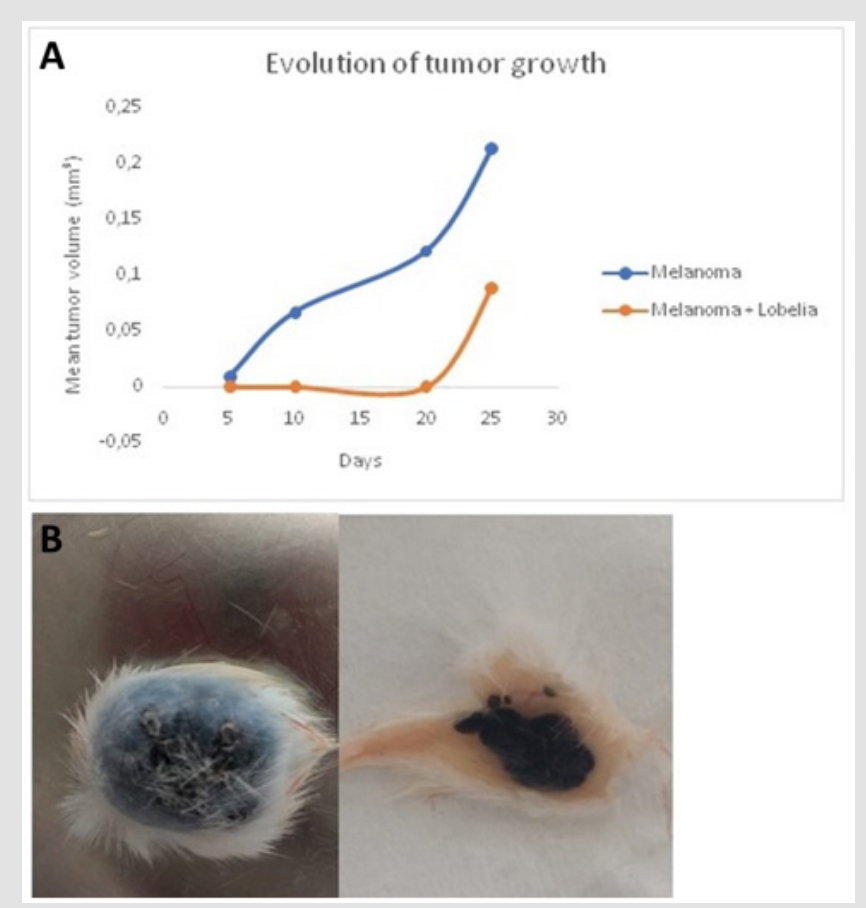

Figure 1: Lobelia inflata reduces tumor growth.

A) Representative graph of tumor growth in the Melanoma group and in the Melanoma + Lobelia group, $\mathrm{p}=0.007$.

B) Representative images of Balb/c mice with non-ulcerated and ulcerated B16F10 melanomas. Note the presence of ulcerations in the melanoma group.

\section{Reduction of Angiogenesis by Lobelia inflata}

Angiogenesis is a central process during cancer development and therefore is considered one of the hallmarks in melanoma. The vascularization of the tumors was compared in the Melanoma and Melanoma + Lobelia groups from the morphometric analyzes.
The results showed a high growth rate of new vessels in the group not treated with Lobelia inflata, presenting a significant difference between the groups (treated and untreated). Lobelia inflata showed a positive impact on melanoma therapy reducing the number of vessels in this experimental model (Figure 2). 


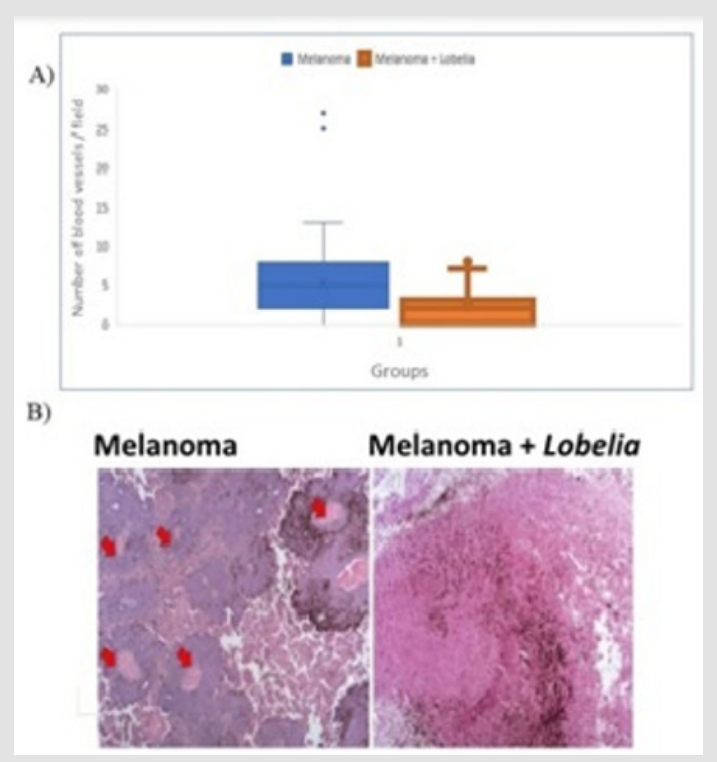

Figure 2:

A) Representative graph of the quantification of the tumors blood vessels number on Melanoma and Melanoma + Lobelia groups in 15 different fields. Melanoma: $\mathrm{n}=7$; Melanoma + Lobelia: $\mathrm{n}=7 ; \mathrm{p}=0.00089$.

B) Representative image of the histological analyses of blood vessels ( $40 \times$ magnification).

Analysis of Cell Proliferation by Tumor Mitotic Index and after Treatment with Lobelia inflata

It is known that among the prognostic factors used to classify melanoma aggressiveness, mitotic index is the most accepted and clinically used. When the two groups were compared, it was found that there was no significant difference in the means of the number of mitoses between the untreated group and the one that received the Lobelia inflata treatment, as shown in Figure 3. This suggests that the presence of the extract did not influence the reduction of the number of mitoses in this experimental model (Figure 3).

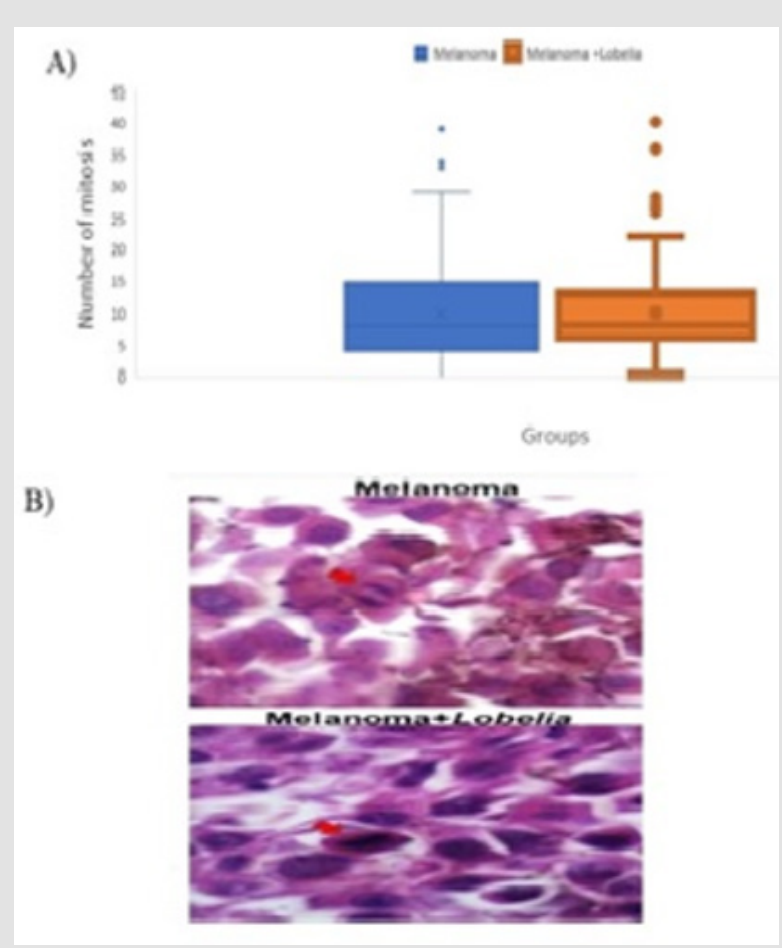

Figure 3:

A) Representative graph of the quantification of mitoses number on Melanoma and Melanoma + Lobelia groups in 15 fields. Melanoma: $\mathrm{n}=7$; Melanoma + Lobelia: $\mathrm{n}=7 ; \mathrm{p}=0.9833$.

B) Representative image of the histological analysis of mitoses (200 $\times$ magnification). 
Analysis of Inflammatory Cells in Melanoma after Treatment with Lobelia inflata

Neutrophils and macrophages were quantified in all animals of both groups (Melanoma and Melanoma + Lobelia). The mean number of neutrophils showed a significant difference between the two groups. As shown in Figure 4, the presence of the extract exerted influence on the neutrophil count, and the group that received it showed a greater dispersion of the data around the mean in relation to the Melanoma group. In this case, the number of neutrophils suggests that acute inflammation was predominant in the group receiving the extract.

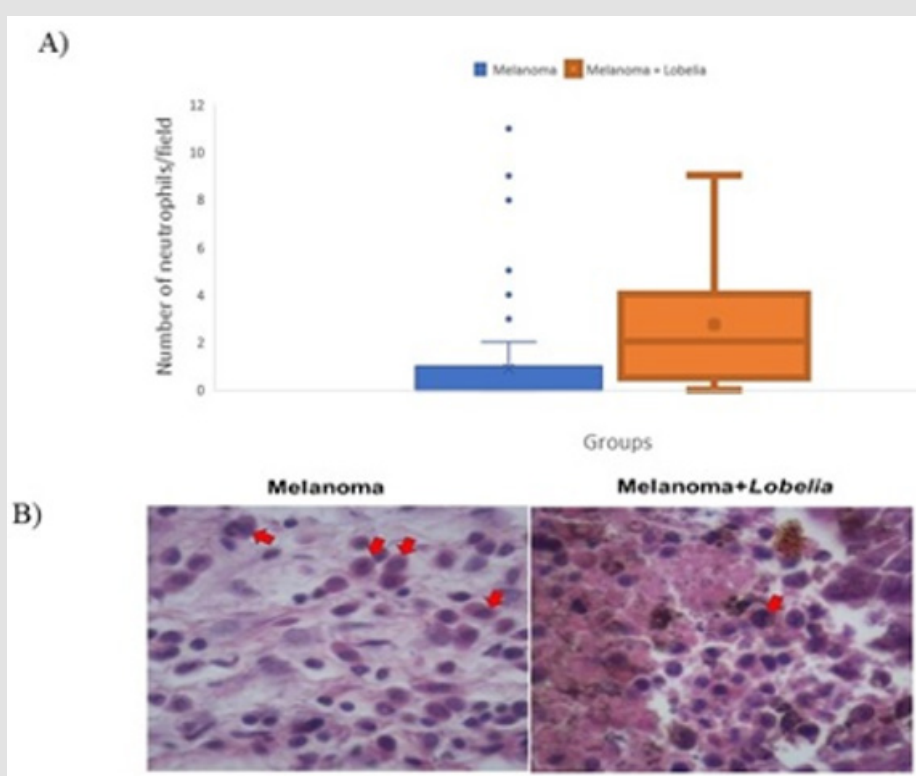

Figure 4:

A) Representative graph of the quantification of neutrophil numbers on Melanoma and Melanoma + Lobelia groups in 15 different fields. Melanoma: $\mathrm{n}=7$; Melanoma + Lobelia: $\mathrm{n}=7 ; \mathrm{p}=0.0001$.

B) Representative image of the histological analysis of neutrophils ( $200 \times$ magnification).

A)

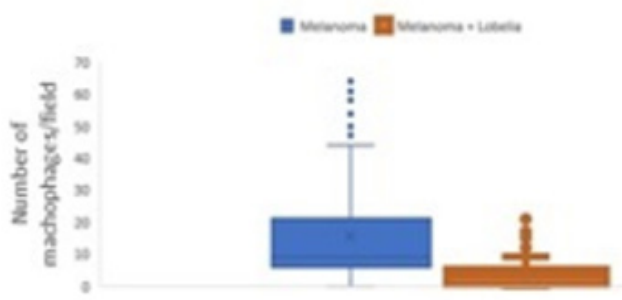

Groups

B)
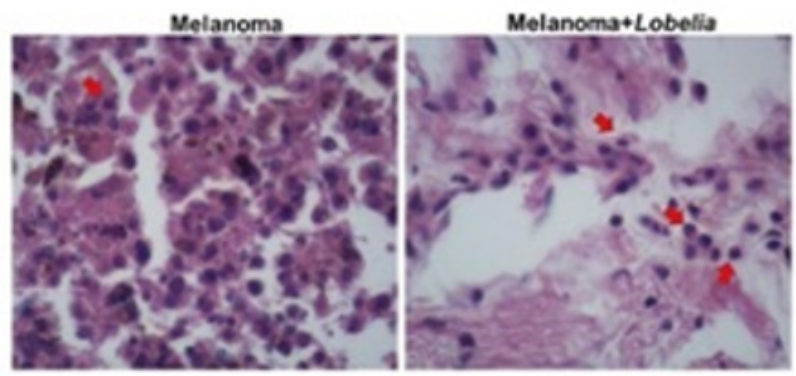

Figure 5:

A) Representative graph of the quantification of macrophage numbers on Melanoma and Melanoma + Lobelia groups in 15 different fields. Melanoma: $\mathrm{n}=7$; Melanoma + Lobelia: $\mathrm{n}=7 ; \mathrm{p}=0.0001$.

B) Representative image of the histological analysis of macrophages (200× magnification). 
The mean number of macrophages showed a significant difference between the two groups. The Melanoma group presented greater variability and higher median value in relation to the other group, which suggests the onset of chronicity of the present inflammatory process, especially in the animals that did not receive the plant extract (Figure 5). In controls groups 01 (PBS) and 02 (Lobelia), no evident morphological alterations could be seen in the skin.

\section{Discussion}

In this study, we used the B16F10 melanoma cells, which is a well validated model for primary tumor. The F10 line of B16 melanoma has been widely used by subcutaneous injection due to its high aggressiveness and also because it has great metastatic potential [9]. Current works have showed the antitumor effect of extract plants in tumorigenesis; however, the role of the Lobelia inflata in the development and progression of melanoma is not fully understood. Here, we bring evidence that the Lobelia inflata can contribute towards a protective role against melanoma progression. Our results showed that the plant extract reduces incidence of ulceration, reduces the tumor growth, diminishing the angiogenesis process and part of the chronic inflammation. Recently, a study that used Lobelia inflata in the same experimental model of melanoma B16F10 published promising results such as the suppression of cell recruitment towards the mouse's peritoneal cavity causing a reduction of circulation leukocytes and, in addition, proved that this extract was able to inhibit the production of pro inflammatory cytokines IL-6, TNF- $\alpha$, IL-1 $\beta$ and significantly reduced tumor growth.

The positive results indicate that Lobelia inflata can be instrument of new researches about anti-tumor herbal treatments [10]. Some studies have demonstrated the antitumor action of Lobelia chinensis, another species of the genus Lobelia, in breast cancer from the isolation of soluble ethanol molecules, which inhibited the proliferation of MCF7 cells, cancer cells from breast cancer cultured and prepared in a biochemical assay. Among the isolated molecules of Lobelia chinensis, polyphenols were found in vast quantities [13]. Polyphenols, for their part, are already recognized for their potent antioxidant properties and their marked effects in the prevention of oxidative stress, which is strongly associated with cancer [14]. Since tumor thickness and ulceration are predictors of survival, according to the American Joint Committee on Cancer (AJCC), it was possible to observe that the Lobelia inflata plant extract had an important impact on the tumor evolution in the model concerning these aspects.

It is rationally to infer that Lobelia inflata had influence in the dynamics of the interaction between normal cells and the tumor, as well as in the elements that determine its microenvironment. It is noteworthy that melanoma presents an early stage of radial growth and its behavior involves less aggressiveness and a later vertical phase, in which the reticular dermis and the subcutaneous tissue are affected, presenting worse prognosis [15]. Therefore, the probable effect exerted by the plant reflects the delayed growth of the tumors in the treated group, besides justifying the smaller dimensions when compared to the untreated animals. Thus, cutaneous melanoma developed in rats that did not receive the extract would present a more aggressive behavior and could be associated with a worse clinical outcome. During all experimental period, only one mouse from the Melanoma group died, which corresponded to $4 \%$, in the total of animals. Angiogenesis is a crucial process during tumor formation and also in the metastatic process, requiring the formation of new vessels so that the cancer cells settle in the target site and maintain a continuous growth.

Invasive capacity is ensured by nutrients, oxygen and by the removal of toxic residues, and thus invasive tumors are a common site of angiogenesis, stimulated by factors such as VEGF (Vascular Endothelial Growth Factor) [16]. Lobelia inflata contains two subfamilies of polyphenols that differ in composition [17]. It is worth mentioning that these compounds manifest wide biological activities, including antibacterial, antioxidant, anti-inflammatory and anticarcinogenic activities [18]. Moreover, angiogenesis is also targeted by polyphenols. These substances have been shown to reduce the migration of end othelial cells and vascular smooth muscle cells, which are essential for the process of blood vessel formation. Besides, these specific polyphenols decrease the expression of angiogenic factors, among them VEGF [19]. Thus, it is suggested that such a relationship between polyphenols and inhibition of angiogenesis is similar to the mechanism responsible for reducing the number of vessels in the treated group, possibly caused by Lobelia. In the initial inflammatory process, the inflammatory cells produce cytokines, growth factors, prostaglandins and leukotrienes, acting as true chemical agents that favor cell reproduction [20].

Upon the interaction of these elements with their receptors, the tumor develops and progresses, stimulating immune responses [21]. However, the immune system can be overcome by tumor cells, since they have a high replicative power, and also due to mutations that promote the inhibition of tumor suppressor genes, the ability to activate proto-oncogenes and the reduction of apoptosis [22]. Moreover, the higher the tumor growth rate, the more it produces inflammation, which, in turn, promotes the tumor's expansion [23]. From these considerations, it is possible to relate the findings concerning the inflammatory process to the greater melanoma aggression and propagation capacity, being important the evaluation of the cell numbers involved in this process. According to the obtained results, the performance of the plant extract may have reflected distinct phases of the inflammation, reducing what would be considered part of a chronic process represented by macrophages, but without reducing the acute inflammation represented, especially by neutrophils. It is known that more than 20 alkaloids represented by active compounds of the Lobelia inflata species have been described, being one of these components, in particular, responsible for acting at the level of central nervous system. 
Among all substances present in the extract, the action of Lobelin, which acts both as an agonist and antagonist at nicotinic acetylcholine receptors, interacts with neurotransmitter transporters including the dopamine and serotonin transporters, and the vesicular monoamine transporter 2 (VMAT2), besides altering the presynaptic dopamine (DA), interfering in the storage and release of the same. Thus, lobeline is the main target of studies related to the Lobelia inflata species, a subject of interest focused on the treatment of drug abuse and Central nervous system disorders, such as Alzheimer's and Parkinson's diseases, attention deficit, restlessness, impulsivity and hyperactivity. Nevertheless, the antitumor effect capable of causing histological changes is something innovative [24]. Therefore, biosynthesis and isolation of lobeline-related alkaloids is still the subject of discussion. Studies in this area involve different and increasingly sophisticated biochemical tests whose purpose is to find new alkaloids and their respective functions.

It is worth mentioning that herbaceous plants have gained space in research and melanoma is an important subject in this field. Another experimental trial using B16F10 implant in Balb/c mouse tested the extract of Arctium lappa and suggested the importance of inflammation in the pathophysiology and progression of this cutaneous cancer. The intraperitoneal plant injection reduced edema formation and suppressed the release of NO (nitric oxide), IL-6, TNF- $\alpha$, and IL-1 $\beta$ in the peritoneal cavity of animals. Besides, the extract was also injected subcutaneously and reduced the size of tumor improving mice survival [25]. Accordingly, our study suggested and sought to understand which mechanisms may have played a role in tumor growth retardation, in reducing neoangiogenesis and in reducing the inflammatory process represented by macrophages, independently of a specific component of the extract. Undoubtedly, further studies are needed in order to discover which of the various alkaloids present in the plant would have been responsible for such findings.

\section{Conclusion}

The present study concluded that Lobelia inflata is a potential therapeutic tool, and its mechanisms of action may in the future mean an effective strategy in the fight against melanoma. The delayed growth of the cutaneous tumor as well as the reduction of the angiogenesis process and part of the chronic inflammation are undoubtedly good predictors for the choice of an effective therapy involving the Lobelia inflata plant extract. As future perspectives a relevant aspect that should be considered is the presence of distance metastases.

\section{Author Contributions}

Conception and design: LCI and CTS; Performed the experiments: LCI, NPG, MSRM, MPC, KFZ, CTS; Formal analysis: all authors; Data interpretation: LCI, KFZ, CTS; Writing-original draft preparation: LCI and CTS; Critical revision of the manuscript: all authors.

\section{Funding}

The research did not receive any external funding.

\section{Acknowledgement}

We thank the prof. Lucila Costa Zinni Angelotti for the statistical analysis, Adair Ferreira de Lima for the preparation of the slides and Vanessa L. Campo for English correction.

\section{References}

1. Meier F, Schittek B, Busch S, Garbe C, Smalley K, et al. (2005) The RAS/ RAF/MEK/ERK and PI3K/AKT signaling pathways present molecular targets for the effective treatment of advanced melanoma. Front Biosci 10: 2986-3001.

2. Weinlich G, Bitterlich W, Mayr V, Fritsch PO, Zelger B (2003) Metallothionein-overexpression as a prognostic factor for progression and survival in melanoma. A prospective study on 520 patients. Br J Dermatol 149(3): 535-541.

3. Moreno, M Conte B, Menegat E (2015) Clinical-epidemiological Differences between Male and Female Patients with Diagnosis of Cutaneous Melanoma in the West of Santa Catarina. Brazilian Journal of Cancerology, Chapecó, Mar 61(1): 15-21.

4. Chabner BA, Longo DL (2015) Harrison's Oncology Handbook. $\left(2^{\text {nd }}\right.$ Edn.). Porto Alegre: AMGH.

5. Nazarian R, Shi H, Wang Q, Kong X, Koya RC, et al. (2010) Melanomas acquire resistance to $\mathrm{B}-\mathrm{RAF}(\mathrm{V} 600 \mathrm{E})$ inhibition by RTK or N-RAS upregulation. Nature 468(7326): 973-977.

6. Ford D, Bliss JM, Swerdlow AJ, Armstrong BK, Franceschi S, et al. (1995) Risk of cutaneous melanoma associated with a family history of the disease. Int J Cancer 62(4): 377-381.

7. Nagananda GS, Krishnamoorthy A, Das A, Bhattacharya S (2012) Phytochemical screening and evaluation of antimicrobial activities of in vitro and in vivo grown plant extracts of Lobelia inflata L 3(3): 433-442.

8. Folquitto DG, Swiech JND, Pereira CB, Bobek VB, Halila GC, et al. (2019) Biological activity, phytochemistry and traditional uses of genus Lobelia (Campanulaceae): A systematic review. Fitoterapia 134: 23-38.

9. Li M, Xu F, Muller J, Hearing VJ, Gorelik E (1998) Ecotropic C-type retrovirus of B16 melanoma and malignant transformation of normal melanocytes. Int J Cancer 76(3): 430-436.

10. Garcia NP, Ireno LC, Castro MP, Reis MS, Gardinassi LG, et al. (2020) Antitumoral Effect of Lobelia inflata in an Experimental Mouse Model of Melanoma. Biomed J Sci \& Tech Res 25(1): 18856-18864.

11. Looney WB, Mayo AA, Janners MY, Mellon JG, Allen P, et al. (1971) Cell proliferation and tumor growth in hepatomas 3924A. Cancer Res 31(6): 821-825.

12. Bono A, Tomatis S, Bartoli C, Tragni G, Radaelli G, et al. (1999) The ABCD system of melanoma detection: A spectrophotometric analysis of the Asymmetry, Border, Color, and Dimension. Cancer 85(1): 72-77.

13. Da Silva CFN, Melo GP, Bernardes SS, Cecchini AA (2013) Experimental models of murine melanoma in vivo. Biosaúde, Londrina 15(2): 73-80.

14.Zhang L, Khoo CS, Koyyalamudi SR, De Pedro N, Reddy N (2017) Antioxidant, Anti-inflammatory and Anticancer Activities of Ethanol Soluble Organics from Water Extracts of Selected Medicinal Herbs and Their Relation with Flavonoid and Phenolic Contents. Pharmacologia 8(2): 59-72. 
15. Dai J, Mumper RJ (2010) Plant phenolics: extraction, analysis and their antioxidant and anticancer properties. Molecules 15(10): 7313-7352.

16. Wainstein AJA, Belfort FA (2004) Conduct for cutaneous melanoma Magazine of the Brazilian College of Surgeons 31(3): 204-214.

17. Pollard JW (2008) Macrophages define the invasive microenvironment in breast cancer. J Leukoc Biol 84(3): 623-630.

18. Biavatti MW (1994) Studies on 8,10-di-n-propyl-lobelidiol: A new alkaloid isolated from Siphocampylus verticillatus (cham.) G. Don., Campanulaceae. Thesis (Dissertation), Federal University of Paraná Curitiba, Brazil.

19. Araújo JR, Gonçalves P, Martel F (2011) Chemopreventive effect of dietary polyphenols in colorectal cancer cell lines. Nutr Res 31(2): 7787.

20. Negrão MRBSSS (2011) Mechanisms of modulation of inflammation and angiogenesis by natural polyphenols. Dissertation of candidacy for the degree of Doctor in Metabolism, Clinic and Experimentation, presented to the Faculty of Medicine of the University of Porto- Medicine Course, University of Porto, Porto, Portugal.

\section{ISSN: 2574-1241}

DOI: $10.26717 /$ BJSTR.2020.26.004348

Cristiane Tefé-Silva. Biomed J Sci \& Tech Res

(c) (9) This work is licensed under Creative

Submission Link: https://biomedres.us/submit-manuscript.php
21. Barreto RDC, Pereira GAS, Da Costa LJ, Cavalcanti HRBB (2011) The Double Role of Inflammation in the Emergence of Carcinogenic Injuries. Brazilian Journal of Health Sciences, Related searches, Apr 14(4): 107114.

22. Maria AG, Dillenburg Pilla P, Reis RI, Floriano EM, Tefé Silva C, et al. (2016) Host kinin B1 receptor plays a protective role against melanoma progression. Sci Rep 6: 22078.

23. Hanahan D, Weinberg RA (2011) Hallmarks of cancer: the next generation. Cell 144(5): 646-674.

24. Kursinszki L, Szőke É (2015) HPLC-ESI-MS/MS of brain neurotransmitter modulator lobeline and related piperidine alkaloids in Lobelia inflata L. J Mass Spectrom 50(5): 727-733.

25. Nascimento BAC, Gardinassi LG, Silveira IMG, Gallucci MG, Tomé MA, et al. (2019) Arctium lappa Extract Suppresses Inflammation and Inhibits Melanoma Progression. Medicines 6(3).

$\begin{array}{ll}\text { BIOMEDICAL } & \text { Assets of Publishing with us } \\ \text { RESEARCHES } & \text { - Global archiving of articles } \\ \text { - Immediate, unrestricted online access }\end{array}$

